\title{
Comparative Analysis of Bacterial and Archaeal Population Structure by Illumina Sequencing of 16S rRNA Genes in Three Municipal Anaerobic Sludge Digesters
}

Munawwar Ali Khan ( $\sim$ Munawwar.Khan@zu.ac.ae)

Zayed University https://orcid.org/0000-0001-5683-300X

Shams Tabrez Khan

Aligarh Muslim University Faculty of Agricultural Sciences

Milred Cedric Sequeira

Zayed University

\section{Sultan Mohammad Faheem}

Manipal Academy of Higher Education

\section{Research}

Keywords: Anaerobic digester, Hydrogenotrophic Methanogens, Thermophiles, Halophiles

Posted Date: August 19th, 2020

DOI: https://doi.org/10.21203/rs.3.rs-60183/v1

License: (c) (i) This work is licensed under a Creative Commons Attribution 4.0 International License. Read Full License 


\section{Abstract}

Understanding the microbial communities in anaerobic digesters is important for better regulation, operation, and sustainable management of the sludge produced at various stages of wastewater treatment processes. Microbial communities in the anaerobic digester of the gulf region where the climatic conditions and other factors may impact the incoming feed have not been documented. Archaeal and Bacterial communities of three full-scale anaerobic digesters, namely AD1, AD3 and AD 5 were analyzed by Illumina sequencing of 16S rRNA genes. Among bacteria, the most abundant genus was fermentative bacteria Acetobacteroides (Blvii28). Other predominant bacterial genera in the digesters included thermophilic bacteria (Fervidobacterium and Coprothermobacter) and halophilic bacteria like Haloterrigena and Sediminibacter. This can be correlated with the climatic condition in Dubai, where the bacteria in the original feed may be thermophilic or halophilic as much of the water used in the country is desalinated seawater. Propionic acid-producing bacteria like Paludibacter and propionate oxidizing bacteria like W5 were also dominating group and were found in all the digesters. The predominant Archaea include mainly the members of phylum Euryarchaeota and Crenarchaeota belonging to genus Methanocorpusculum, Metallosphaera, Methanocella, and Methanococcus. The highest population of Methanocorpusculum (more than $50 \%$ of total Archaea) hydrogenotrophic archaea matches with the high population of Acetobacteroides (Blvii28) and Fervidobacterium bacteria which ferments the organic substrates to acetate and $\mathrm{H} 2$. Coprothermobacter, which is known to improve protein degradation by establishing a syntrophy with hydrogenotrophic archaea, was also one of the dominant genera in the digesters. This study, for the first time, contributes to an in-depth understanding of the phylogenetic diversity of a microbial community of three full-scale anaerobic digesters of a municipal wastewater treatment plant in Dubai, UAE.

\section{Introduction}

The process of anaerobic digestion is a multistep microbial process that includes the microbial breakdown of organic matter to produce $\mathrm{CO}_{2}, \mathrm{CH}_{4}$, and $\mathrm{H}_{2} \mathrm{O}$ by a complex microbial community, including archaea and bacteria. These products can be used as biogas, which can be further processed to generate electricity or fuel for transportation[1, 2]. The energy obtained from the anaerobic digesters can also be used for the operation of the wastewater treatment plant itself [3]. Due to these possible applications of the gases and the rising cost of conventional fossil fuels, the anaerobic digestion process is emerging as one of the most sustainable methods for the management of organic waste [4]. The process is already being used for the generation of renewable energy in many countries worldwide [5]. Besides, anaerobic digestion is of environmental significance also as it helps in managing wastes and reducing greenhouse gasses [6]. But there are clear challenges in translating the technology for simple commercial applications and general use [7]. One of the reasons is the complexity of the microbial community involved in the process, making it difficult to understand the specific roles played by different bacteria and their maintenance as consortia $[8,9]$. Therefore, it is essential to understand the roles played by various microorganisms in the process of anaerobic digestion.

During the conversion of organic wastes into $\mathrm{CH}_{4}$ gas, several microbial processes are involved [10]. It consists of following necessary steps, breakdown of complex organic molecules to simple organic compounds, followed by the conversion of these simple organic molecules to organic acids (acidification), and finally, the transformation of these organic acids into the $\mathrm{CH}_{4}$ gas. Methanogens (methane-producing bacteria) also are of two major types, namely, hydrogenotrophic and acetolactic, depending on the type of substrate they utilize for the $\mathrm{CH}_{4}$ production. Each of these processes is carried out by different microorganisms in the presence of various other unrelated microorganisms. But the complex interplay between these microorganisms may influence these processes adversely 
or favorably. Multiple factors influence the composition of the microbial community present in the anaerobic digesters[11]. One crucial factor can be the microbial community in the feed of the anaerobic digester, which can finally influence the microbial community in the anaerobic digester $[12,13]$. The composition of the microbial community in the feed can also change with environmental conditions as it has been demonstrated earlier also that the environmental conditions affect the type of methanogens present in anaerobic digesters [14].

Nowadays, next-generation sequencing-based on 16S rRNA amplicon is commonly applied for an in-depth understanding of microbial community structure and dynamics in a wide variety of engineered anaerobic digester systems [15-17]. The Illumina is a promising high throughput sequencing platform to illustrate the taxonomic and functional analysis of diverse microbial populations in various environmental samples. There are several studies conducted for the phylogenetic classification of microbial communities in full-scale anaerobic sludge systems worldwide; however, next-generation sequencing has not been applied to study the microbial community structure in anaerobic sludge digester of full-scale municipal wastewater treatment plants in the UAE. Although we examined the anaerobic digester microbial community using fluorescent in situ hybridization and quantitative PCR technique, a comprehensive mapping of key microbial operational taxonomic groups was necessary [18]. The sewage treatment plant, located in Jebel Ali, is one of the major wastewater treatment plants in Dubai, UAE. Its efficient operation and maintenance are indispensable for the city of Dubai.

Furthermore, as per our knowledge, a deeper understanding of microbial community structure of full-scale anaerobic sludge digesters of municipal sewage treatment plants in the UAE using high throughput Illumina sequencing of 16S rRNA gene has not been attempted. Despite well-documented studies of the influence of physicochemical operational parameters on microbial composition, this study does not focus on the effect of operational parameters for anaerobic digestion on the microbial population. It was also because all three digesters operated under stable operating conditions fed by the wastewater sludge produced at various stages of the treatment of wastewater. Therefore, in this context, this investigation focussed on the comparison of obtained taxonomic results of the dominant genera of the bacterial and archaeal community structure of three full-scale anaerobic digesters involved in the critical steps of anaerobic digestion process as determined by the high-throughput Illumina sequencing approach. The present study hypothesized that the anaerobic digester microbial community performing under stable physicochemical conditions and sludge feed should have a core functional taxonomic group with a welldefined role in each phase of the cycle of anaerobic digestion.

This study also attempts to find out the relative abundance of a specific taxonomic group between three types of the anaerobic digester and within the same digester sample taken at varying periods using Illumina sequencing of 16S rRNA genes.

\section{Materials And Methods}

\subsection{Sampling}

The waste sludge samples were collected from three anaerobic digesters of the Jebel Ali Sewage Treatment Plant (JASTP), UAE. The JASTP is the largest tertiary wastewater treatment infrastructure in Dubai, which serves a population of approximately 3.37 million and process $375,000 \mathrm{~m}^{3}$ of wastewater per day. All the three digesters had a capacity of $7433 \mathrm{~m}^{3}$ and were operating at a mesophilic temperature ranging between $32-37^{\circ} \mathrm{C}$ [19]. The digesters were fed with 60 and $40 \%$ of raw and activated sludge, respectively. Details on the configuration and characteristics of the digesters are listed in Table 1. The samples from the anaerobic digesters were collected in 
autoclaved plastic bottles, and the bottles were transported to the lab on ice at $4{ }^{\circ} \mathrm{C}$ within an hour. The samples collected from three anaerobic digesters over a period of six months were designated as AD1, AD3, and AD5. The collected samples were immediately mixed with $100 \%$ ethanol in a ratio of $1: 1(\mathrm{v} / \mathrm{v})$ and stored at $-20^{\circ} \mathrm{C}$ until DNA extraction. Temperature, $\mathrm{pH}$, and electrical conductivity (EC) were measured in sludge samples at the time of collection using HORIBA U-50 Multi Water Quality Checker (HORIBA Instruments Incorporated, USA).

Table 1

Characteristics and configuration of anerobic digestors

\begin{tabular}{|c|c|c|c|}
\hline \multirow[t]{2}{*}{ Characteristics of digester } & \multicolumn{3}{|c|}{ Digester number } \\
\hline & AD1 & AD3 & AD5 \\
\hline Type of digester & $\operatorname{CSTR}^{\mathrm{a}}$ & CSTR & CSTR \\
\hline Operating temperature & mesophilic & mesophilic & mesophilic \\
\hline \multirow[t]{2}{*}{ Input feed } & $\mathrm{RS}^{\mathrm{b}}-60 \%$ & RS-60\% & RS-60\% \\
\hline & $A S^{c}-40 \%$ & AS-40\% & AS- $40 \%$ \\
\hline Digester capacity $\left(\mathrm{m}^{3}\right)$ & 7433 & 7433 & 7433 \\
\hline Digester feeding rate ( $\mathrm{m}^{3} /$ day) & 2248 & 2148 & 2552 \\
\hline Solid retention time (Days) & 16 & 16 & 16 \\
\hline Hydraulic Retention time (Days) & 3.3 & 3 & 2.91 \\
\hline Upflow Velocity (m³/hr) & 120 & 120 & 120 \\
\hline Organic loading rate $\left(\mathrm{Kg} . \mathrm{ODS} / \mathrm{m}^{3} . \mathrm{d}\right)$ & 6.84 & 5.84 & 6.61 \\
\hline
\end{tabular}

\subsection{Genomic DNA extraction}

Genomic DNA was extracted in triplicate for each sludge digester sample using Power Soil DNA Extraction Kit (MO BIO Labs. Inc., Solana Beach, CA). The samples were vortexed and were then centrifuged at 4000 rpm for 5 minutes. DNA was then extracted from $0.25 \mathrm{~g}$ of pellet obtained according to the manufacturer's protocol. The extracted DNA was stored at $-20^{\circ} \mathrm{C}$ until further use. DNA concentration and purity were checked using the Qubit fluorometer (Thermo Fisher Scientific, USA).

\subsection{Illumina sequencing of samples}

The diversity of bacterial and archaeal communities in the samples was determined by amplifying the V3-V4 regions of bacterial and archaeal $16 \mathrm{~S}$ ribosomal RNA (rRNA) genes. The following is a brief description of the main steps and protocol. The extracted genomic DNA samples quality check was performed by quantification using Qubit DNA BR Assay kit (Thermo Fisher Scientific, USA Cat\#Q32853). For the generation of 16S amplicon, the extracted DNA samples were diluted to $10 \mathrm{ng}$ and were amplified for $16 \mathrm{~S}(\sim 1500 \mathrm{bp})$ using $16 \mathrm{~S}\left(5^{\prime}\right.$ AGAGTTTGATCCTGGCTCAG 3') and 16S reverse primers (5' GGTTACCTTGTTACGACTT 3') and positive control (internal metagenomic DNA sample) and no template control. These amplicons were checked on $1 \%$ agarose gel. To 
generate V3-V4 amplicon, 16S amplicon was used as a template with all the samples subjected to V3-V4 amplification ( 460 bp) using V3-V4 forward and V3-V4 reverse primers (primer sequences V3-V4-Forward 5' CCTACGGGNGGCWGCAG 3' and V3-V4 Reverse 5' GACTACHVGGGTATCTAATCC 3') and a positive control (internal metagenomic DNA sample) and without template control [20]. The amplicons were checked on $1 \%$ agarose gel. The V3-V4 amplicons were then cleaned using AMPure XP beads (Beckman Coulter, CA, USA, Cat\# A63882) to get rid of non-specific fragments. The V3-V4 products were used for DNA library preparation using NEBNext Ultra DNA Library Prep Kit for Illumina (New England Biolab, UK, Cat\# E7370L). First, the amplicons were end-repaired and monoadenylated at 3 ' end in a single enzymatic reaction. Next, NEB hairpin-loop adapters are ligated to the DNA fragments in a T4-DNA ligase-based reaction. Following ligation, the loop containing Uracil is linearized using USER Enzyme (a combination of UDG and Endo VIII), to make it available as a substrate for PCR based indexing in the next step. During PCR, barcodes were incorporated using unique primers for each of the samples, thereby enabling multiplexing. The prepared libraries were checked for fragment distribution using D1000 Screen Tapes (Cat\# 50675582, Agilent, CA, USA) and reagents (Cat\# 5067-5583, Agilent, CA, USA). The obtained libraries were pooled and diluted to final optimal loading concentration before cluster amplification on Illumina flow cell. Once the cluster generation is completed, the clustered flow cell is loaded on Illumina HiSeq2500 instrument (Illumina, Inc., San Diego, USA) for amplicon sequencing to generate $0.5 \mathrm{M}, 250$ bp paired-end reads per sample using pair-end approach.

\subsection{Bioinformatics analysis}

The schematic bioinformatics analysis pipeline is represented in supplementary Fig. 1. Briefly, the following steps were involved in the bioinformatics analysis for further processing of the reads and quality filtering sequencing data. The quality checking of the raw fastq files was carried out using FASTQC to check for base quality, base composition and G.C. content. Based on the quality report of fastq data, the sequence reads were trimmed using fastq-mcf where necessary only to retain high-quality sequences for further analysis. Besides, the low-quality sequence reads were excluded from the analysis.

The 16S rRNA gene identification was conducted by identification of the amplicons from the raw fastq files based on the presence of conserved region forward and reverse. The paired-end sequences read contained a spacer region, some portion of the conserved region, and V3-V4 region. As a first step, the spacer and conserved regions were removed from paired-end reads. After trimming the unwanted sequences from original paired-end data, a consensus V4 region sequence was constructed using Fast Length Adjustment of SHort reads (FLASH V1.2.7) described by[21].

The pre-processing of reads was done by dereplication step. The dereplication is the identification of unique sequences so that only one copy of each sequence is reported. USEARCH is used for dereplication with the derep_full length. Chimeric sequences are formed as a result of experimental artifact, which is a fusion between two or more original sequences. UCHIME utility from usearch was used to remove the chimeras using the de novo approach[22].

The operational taxonomic unit (OTU) identification and singleton removal was done by performing the following steps. The sequences having a similarity of $97 \%$ were grouped using a closed reference method under a single operational taxonomic unit (OTU) for classification of the whole data. Any OTU that has a count of 1, i.e., only one sequence present once in a single sample (singleton) were filtered out as these may have been created as an experimental artifact rather than being some novel organism. For taxonomic classification, one representative sequence from each OTU was picked and classified using the RDP classifier against the green gene database at $97 \%$ similarity. The taxonomy classification was done at phyla, order, family, and genera level. The raw sequences 
have been deposited in National Center for Biotechnology Information (NCBI Bethesda MD, 20894 USA) under BioProject accession number PRJNA602372.

\subsection{Statistical analysis}

In the present study, two types of comparisons are carried out. Firstly, intergroup comparisons were carried out to find differences in genera across each of the groups as follows: AD1 v/s AD3; AD3 v/s AD5 and AD1 v/s AD5. The comparisons are performed using a t-test, and any genus having a p-value less than 0.05 in the t-test is considered as differentially present. Secondly, intragroup comparisons are carried out for each of the three groups, between samples collected at a different period. As these comparisons are between a pair of samples (single sample comparison) without any replicate, a different approach is taken to identify the differential genera. First, the unique genera for both the samples are listed out separately. Then, a ratio of relative abundance for both the samples are calculated for common genera between the samples. These ratios are then converted into log scale to get a normal distribution, and any genera having a value beyond mean $\pm 2 \mathrm{SD}$ are considered as differentially present genera.

\section{Results And Discussion}

\subsection{Physicochemical conditions of Anaerobic digesters}

The physicochemical conditions of the digester operation are given in Table 2. All three digesters were operated in almost similar physicochemical conditions, i.e., under mesophilic temperature $\left(34^{\circ} \mathrm{C}\right)$, neutral $\mathrm{pH}$ range of $7.13-$ 7.55. At the same time, dry solids, volatile solids concentrations varied respectively, between 2.56 to $5.74 \%$ and 43.75 to $70.95 \mathrm{mg} \mathrm{L}^{-1}$. Among the three digesters, however, AD3 had a relatively low organic loading rate of $5.84 \mathrm{~kg}$. ODS $/ \mathrm{m}^{3}$. d compared to AD1 (6.84 kg. ODS $/ \mathrm{m}^{3}$. d) and AD5 (6.61 kg. ODS/m³. d). The electrical conductivity (EC) of all sludge digestors found to be towards the higher range. The highest conductivity of sludge samples was found for AD5 (12.33-15.46 mS cm-1) followed by AD3 (11.71-14.44 $\mathrm{mS} \mathrm{cm}^{-1}$ ), and AD1(9.77-13.45 mS cm-1). The EC value range found in this study was similar to a study conducted recently in Austria in which the effects of various co-substates were evaluated for the microbial community composition of seven full-scale anaerobic digesters fed with or without co-substrates [17]. Overall, three anaerobic digesters showed acceptable levels of main operational parameters and were performing stably during the sampling period. 
Table 2

Physicochemical parameters of anaerobic digesters samples

\begin{tabular}{|c|c|c|c|c|c|c|c|c|c|}
\hline Digester & $\begin{array}{l}\text { Digester } \\
\text { sample }\end{array}$ & $\begin{array}{l}\text { Temperature } \\
\left({ }^{\circ} \mathrm{C}\right)\end{array}$ & $\mathrm{pH}$ & $\begin{array}{l}\mathrm{EC} \\
(\mathrm{ms} \\
\mathrm{cm}^{-} \\
1)\end{array}$ & $\begin{array}{l}\text { Dry } \\
\text { solids } \\
\text { (\% } \\
\text { [wt/vol]) }\end{array}$ & $\begin{array}{l}\text { Volatile } \\
\text { solids } \\
\text { (\% } \\
\text { [wt/wt] } \\
\text { of TS) }\end{array}$ & $\begin{array}{l}\text { Volatile } \\
\text { fatty } \\
\text { acids } \\
\text { (mg } \\
\text { HAC/L) }\end{array}$ & $\begin{array}{l}\text { Dissolved } \\
\text { sulfide } \\
\text { (mg/L) }\end{array}$ & $\begin{array}{l}\text { Alkalinity } \\
\text { (mg/L) }\end{array}$ \\
\hline \multirow[t]{3}{*}{ AD1 } & $A D 1 N$ & 34.3 & 7.33 & 10.77 & 3.34 & 70.27 & 177 & 30.6 & 3249 \\
\hline & AD1D & 33.8 & 7.13 & 13.45 & 2.91 & 70.56 & 165 & 37.2 & 3014 \\
\hline & $\mathrm{AD} 1 \mathrm{~F}$ & 33 & 7.33 & 9.77 & 4.34 & 70.95 & 195 & 38 & 3451 \\
\hline \multirow[t]{3}{*}{ AD3 } & AD3S & 34.5 & 7.22 & 14.44 & 2.56 & 43.75 & 153 & 34.40 & 3390 \\
\hline & AD3D & 33.5 & 7.27 & 11.71 & 3.35 & 70.15 & 168 & 32.40 & 2992 \\
\hline & AD3J & 34.2 & 7.55 & 13.76 & 5.74 & 43.76 & 205 & 37.20 & 3512 \\
\hline \multirow[t]{4}{*}{ AD5 } & AD5S & 34.4 & 7.36 & 12.33 & 2.79 & 50.3 & 150 & 16.80 & 3190 \\
\hline & AD5N & 33.8 & 7.45 & 13.75 & 3.41 & 56.52 & 145.5 & 27.60 & 2893 \\
\hline & AD5J & 32.9 & 7.46 & 14.39 & 3.35 & 54.54 & 195 & 26.40 & 3857 \\
\hline & AD5F & 33.4 & 7.50 & 15.46 & 3.54 & 67.49 & 191 & 47.20 & 3498 \\
\hline
\end{tabular}

\subsection{Illumina sequencing of 16S rRNA genes}

To check the quality of Illumina sequencing results, the number of raw reads, total data obtained, and the average base quality for each sample are shown in supplementary Fig. 2. It can be observed that all the samples, except two of them, have an average Phred score of more than 30. To retain only the high-quality data reads with a Phred score $>$ Q30 is taken for down-stream analysis. The total number of raw reads of 16S rRNA genes identification, and CR (conserved region for V3-V4) trimmed reads are presented in supplementary Fig. 3. Overall, 3340 MB sequencing data were generated using Illumina sequencing, revealing $1.6 \times 10^{6}$ raw reads. Using quality filtering, $60 \%$ of the total reads were removed, resulting in an average of $0.6 \times 10^{6}$ reads per sample. A total of 419 bacterial community taxa found and classified at Phyla (33), Class (64), Order (99), Family (116), and Genera level (107). To find out the major taxa in each level, filtration is applied to retain taxa, which are present at least $1 \%$ in any of the samples, resulting in 84 OTUs based on $97 \%$ similarity. While $6.1 \pm 1.24 \%$ reads were assigned to Archaea, which included five major phyla, 83 Genera, and 152 species. Bacteroidetes, Firmicutes, Synergistetes, and Theromotogate, OP8, and Chloroflexi were found to be dominant in all the digesters, accounting for $90.85 \%$ of all sequences (Fig. 1). In this study, Bacteroidetes were found to be the most abundant phylum (41.44\%), followed by Firmicutes (23.42\%), Synergistetes (7.22\%), Thermotogae (6.70\%), and Chloroflexi(6.69\%) and OP8 (5.3\%). Other phyla, detected at percentages $>1 \%$ were affiliated to Actinobacteria (3.1\%), Proteobacteria (1.4\%), and WWE1 (1.21\%). The overall abundance of bacterial community phyla observed in this study is similar to closely related studies where a group of Phyla, namely Chloroflexi, Firmicutes, and Bacteroidetes were found to be dominant in full-scale anaerobic sludge digester samples $[17,23,24]$. However, unlike these studies, the percentages of Bacteroidetes $(44 \%)$ detected in this study were significantly high and the population of proteobacteria was very low (1.4\%). The higher population of Bacteroidetes phyla can be associated with high hydrolytic activity in full-scale anaerobic digesters[25].

\subsection{Bacterial community diversity at genus level}


The rarefaction curves show that among the three digesters, the highest diversity was present in AD5. At the same time, the least diverse microbial community was found in AD1 (Fig. 2A). Fifty-one genera were detected as core genera in all the digesters, including Acetobacteroides (Blvii28), Coprothermobacter, Fervidobacterium, Clostridium, Caldilinea, Allochromatium, Sediminibacter, and T78 (Fig. 2B). In addition to these genera, many of the bacteria were identified as unknown bacteria. Most of these genera are associated with an anaerobic digester, some have been reported from anaerobic digester earlier also[11]. Notably the highest population of genus Acetobacteroides (Blvii28) was present in all the digesters. The cultured representative member of the genus is known to produce acetate, $\mathrm{H}_{2}$, and $\mathrm{CO}_{2}$ as the product of fermentation [26]. Intra-group comparison of three AD3 samples collected between September 2016 and January 2017 showed that the concentration of Blvii28 increased significantly in samples from September 2016 to December 2016 and January 2017 (supplementary file 2). A similar trend was observed for intragroup comparison of AD5 samples collected between September 2016 and January 2017 (supplementary file 3). In contrast, T78 is possibly metabolizing carbohydrates and alcohol via syntrophic interactions [27]. T78 is the member of the phylum Chloroflexi often found to be abundant in full-scale mesophilic anaerobic digesters receiving excess sludge from wastewater treatment plants[28]. Intragroup comparison of AD5 (Jan-Feb 2017) samples found nine-fold increase in concentration of T78 in February 2017 sample compared to January 2017. Many genera found predominantly in the digesters were thermophilic or halophilic. Like, Coprothermobacter is a known proteolytic anaerobic thermophilic bacteria found in many thermophilic anaerobic digester [29]. This genus can also improve protein degradation by establishing a syntrophy with hydrogenotrophic archaea [29]. Other thermophilic bacteria found in the anaerobic digester include Fervidobacterium and Caldilinea, that ferment carbohydrates to lactate, acetate, hydrogen, and carbon dioxide [30, 31].

In comparison, genus Sediminibacter which was initially isolated from marine sediment, was also found as one of the dominant genera [32]. This can be due to the simple reason that most of the water used in Dubai is obtained from the sea and is being used after desalination [33,34]. The incoming feed may contain elevated levels of salts, or it may be due to the fact that Sediminibacter has a unique light-driven sodium ion pump, which helps in their survival in marine habitats [35]. Several purple sulfur bacteria like Allochormatium and Thermotogales AUTHM297 were also part of the microbial community in these digesters. Many sulfate-reducing bacteria were also found, including Desulfomicrobium and Desulfobacter, as the two most dominant genera of SRB. In our previous work using FISH technique, we found that in all three digesters members of the genus Desulfobacter and Desulfobacterium were consistently present in large numbers[19]. It is interesting to note that Desulfomicrobium is also known to be associated with the marine habitat [36].

The process of anaerobic digestion involves the degradation of organic compounds to simple organic compounds, which is most likely carried out by the members of the phylum Bacteroidetes, Firmicutes, Proteobacteria, Synergistetes, and Thermotogae. Other dominant phyla in the fermenters are shown in Fig. 3. Previous studies also demonstrate the presence of these phyla in anaerobic digester as macromolecules degrading bacteria[11]. Mycobacterium, which is associated with the production of Lipases and Lipolytic activity, is also found as one of the predominant genera. Another critical process of anaerobic digestion is acidogenesis, wherein bacteria convert organic monomers into acids like acetic, propionic, and butyric acids. The population of Acidogenic bacteria like Acetobacteroides, Fervidobacterium, Clostridium, and Paludibacter producing acetate lactate or propionate was high in the digesters as reported above. The conversion of these organic molecules to $\mathrm{CH}_{4}$ is carried out mainly by Archaea. However, some bacteria may influence the production of methane. For example, acetate-utilizing uncultured bacteria of Synergistes group 4 and its competition with aceticlastic methanogen [37]. 
The boxplots of intergroup comparisons for differentially present genera among three anaerobic digestor groups (AD1 v/s AD3; AD3 v/s AD5 and AD1 v/s AD5) are shown in Fig. 4 and supplementary file. Three genera Rhodobacter, Allochromatium, and Bifidobacterium, are found to be differentially present across the two groups AD1 and AD3, but among them, only Bifidobacterium showed differential abundance between AD3 and AD5. For the third Comparison (AD1 v/s AD5), genera Propionicimonas, Clostridium, and Paracoccus showed differential abundance between the two groups AD1 and AD5. To find out if the samples showed an overall group-specific trend, a principal component analysis based on all the genera detected is performed. The PC1 vs PC2 PCA plot (Fig. 5) showed no group-specific signature. Overall, from the taxonomic classification at the genera level, it can be observed that different digesters alter the microbiome profile differently. For intergroup comparisons, no abundant genera (present at least $1 \%$ in any of the samples) showed significant differentiation.

\subsection{Archaeal community diversity at genus level}

When analyzed at the genus level, the highest diversity was observed in digester AD5, followed by AD1 and AD3 (Fig. 6A). This was evident from the highest Shannon index obtained for AD1, followed by AD3 and AD5. The predominant Archaea in the three digesters were the members of the phylum Euryarchaeota followed by Crenarchaeota. The population of hydrogenotrophic archaea was higher in the digesters. Methanocorpusculum alone constitutes more than $50 \%$ of the total population of Archaea. Other predominant genera of archaea include Metallosphaera, Methanocella, Methanococus, Acidianus, Natronobacterium, and others shown in Fig. 6B. Methanocorpusculum is one of the hydrogenotrophic methanogens, which was isolated for the first time from the biodigester of the wastewater treatment plant [38]. In contrast, the second most predominant genus was Metallosphaera, which is an extreme thermoacidophile with optimal growth at $74{ }^{\circ} \mathrm{C}$ and $\mathrm{pH} 2.0$ [39]. Methanocella, which was the third most dominant genus, is a mesophilic, hydrogenotrophic methanogen [40]. While Methanococcus is also a thermophilic hydrogenotrophic methanogen and at least one species of the genus is also known to fix nitrogen[41].

It is clear from the microbial community analysis that a core microbial community exists in the anaerobic digesters studied. However, the degradation is carried out by the members of various phylum reported earlier also from the anaerobic digesters. These include the members of the phylum Bacteroidetes, Firmicutes, Proteobacteria, Synergistetes, and Thermotogae [13]. While several genera mainly involved in the conversion of these organic substrates into acetate, butyric acid, propionate, and $\mathrm{H}_{2}$ were found to be the dominant genera in the anaerobic digesters. However, Euryarchaeota, mainly the hydrogenotrophic methanogens, were found as predominant members in the digesters (Fig. 6). The predominant hydrogenotrophic methanogens activities compared to aceticlastic ones, is in agreement with another study[25]. Overall, abundant microbial genera observed in this study, and their predicted role in anaerobic digestion associated with a specific process is shown in Fig. 7. Although the digesters were operated under mesophilic conditions, many thermophilic genera of bacteria and archaea were found to predominate in the digester. It may be because of the presence of these bacteria in the incoming feed. Whether or not these bacteria are initially present in the feed is a matter of further investigation. Furthermore, the bacteria of marine origin like Sediminibacter and Haloterrigena as one of the predominant genera maybe because most of the water used in Dubai is desalinated seawater. The high salinity range suggested indirectly by higher electrical conductivity values observed in this study may be the reason for the occurrence of halophilic bacteria found in anaerobic digester systems.

\section{Conclusions}


In this study, using Illumina sequencing of 16S rRNA genes, we compared bacterial and archaeal community structure of three full-scale anaerobic sludge digesters of a municipal sewage treatment plant in Dubai, UAE. Bacteroidetes, Firmicutes, Synergistetes, Theromotogate, OP8, and Chloroflexi were dominant bacterial community phyla in all the digesters and the highest diversity observed in AD5 followed by AD3 and AD1. The predominant archaea community included mainly the members of phylum Euryarchaeota and Crenarchaeota belonging to genus Methanocorpusculum, Metallosphaera, Methanocella, and Methanococcus with highest archaeal community diversity observed in AD5 as compared to AD3 and AD1. The findings of this study indicate that the predominance of thermotolerant and halotolerant bacteria and archaea in the anaerobic digesters, and this could be due to the influence of environmental conditions on the incoming feed sludge. The anaerobic digesters also characterized by a high population of bacteria known to ferment organic substrates to acetate and $\mathrm{H} 2$ and hydrogenotrophic archaea. This indicates the possible mechanism of $\mathrm{CH} 4$ production by the microbial community present in these digesters. From the taxonomic classification at the genera level, it can be observed that different digesters alter the microbiome profile differently. For intergroup comparisons, no abundant genera (present at least $1 \%$ in any of the samples) showed significant differentiation. In conclusion, a core and the stable, functional microbial community was observed under more or less similar physicochemical conditions.

\section{Declarations}

\section{Availability of data and materials}

The raw sequences have been deposited in National Center for Biotechnology Information (NCBI Bethesda MD, 20894 USA) under Bio-Project accession number PRJNA602372.

\section{Competing interests}

The authors declare they have no competing interests.

\section{Funding}

This work was supported by Zayed University Microbial Diversity and Ecogenomics Research Cluster Project (Grant code: R16092).

\section{Authors' contributions}

MAK and STK contributed equally to data analysis, preparation, and final editing of the manuscript. MAK and SRK are joint corresponding author of the manuscript. MCS collected the samples, prepared genomic DNA, and carried out sequencing. SMF helped in supervision and literature review. All authors read and approved the final manuscript.

\section{Acknowledgements}

The authors wish to thank Jebel Ali Sewage Treatment Plant authority for providing samples and operational parameters data of anaerobic digesters used in this study. 


\section{References}

1. Angelidaki I, Ellegaard L, Ahring BK. Applications of the anaerobic digestion process. Biomethanation II. Springer; 2003, p. 1-33.

2. Kirschke $S$, Bousquet $P$, Ciais $P$, Saunois M, Canadell JG, Dlugokencky EJ, et al. Three decades of global methane sources and sinks. Nature geoscience 2013;6(10):813-23.

3. Silvestre G, Fernández B, Bonmatí A. Significance of anaerobic digestion as a source of clean energy in wastewater treatment plants. Energy Conversion and Management 2015;101:255-62.

4. Lin L, Xu F, Ge X, Li Y. Improving the sustainability of organic waste management practices in the food-energywater nexus: A comparative review of anaerobic digestion and composting. Renewable and Sustainable Energy Reviews 2018;89:151-67.

5. Bond T, Templeton MR. History and future of domestic biogas plants in the developing world. Energy for Sustainable Development 2011;15(4):347-54.

6. Paolini V, Petracchini F, Segreto M, Tomassetti L, Naja N, Cecinato A. Environmental impact of biogas: A short review of current knowledge. Journal of Environmental Science and Health, Part A 2018;53(10):899-906.

7. Nizami DA-S, Saville BA, Maclean H. Anaerobic digesters: perspectives and challenges. 2013, p. $139-51$.

8. Vanwonterghem I, Jensen PD, Ho DP, Batstone DJ, Tyson GW. Linking microbial community structure, interactions and function in anaerobic digesters using new molecular techniques. Current Opinion in Biotechnology 2014;27:55-64.

9. Muvhiiwa R, Hildebrandt D, Chimwani N, Ngubevana L, Matambo T. The impact and challenges of sustainable biogas implementation: moving towards a bio-based economy. Energy, Sustainability and Society 2017;7(1):20.

10. Meegoda JN, Li B, Patel K, Wang LB. A Review of the Processes, Parameters, and Optimization of Anaerobic Digestion. Int J Environ Res Public Health 2018;15(10):2224.

11. Ju F, Lau F, Zhang T. Linking microbial community, environmental variables, and methanogenesis in anaerobic biogas digesters of chemically enhanced primary treatment sludge. Environmental science \& technology 2017; 51(7):3982-92.

12. De Vrieze J, Christiaens MER, Walraedt D, Devooght A, ljaz UZ, Boon N. Microbial community redundancy in anaerobic digestion drives process recovery after salinity exposure. Water Research 2017;111:109-17.

13. Svensson K, Paruch L, Gaby JC, Linjordet R. Feeding frequency influences process performance and microbial community composition in anaerobic digesters treating steam exploded food waste. Bioresource Technology 2018;269:276-84.

14. Karakashev D, Batstone DJ, Angelidaki I. Influence of Environmental Conditions on Methanogenic Compositions in Anaerobic Biogas Reactors. 2005;71(1):331-8.

15. Shin SG, Koo T, Lee J, Han G, Cho K, Kim W, et al. Correlations between bacterial populations and process parameters in four full-scale anaerobic digesters treating sewage sludge. Bioresource Technology 2016;214:711-21.

16. Sposob M, Moon H-S, Lee D, Kim T-H, Yun Y-M. Comprehensive analysis of the microbial communities and operational parameters of two full-scale anaerobic digestion plants treating food waste in South Korea: seasonal variation and effect of ammonia. Journal of Hazardous Materials 2020:122975.

17. Walter A, Probst M, Franke-Whittle IH, Ebner C, Podmirseg SM, Etemadi-Shalamzari M, et al. Microbiota in anaerobic digestion of sewage sludge with and without co-substrates. Water and Environment Journal 2019;33(2):214-22.

Page $11 / 19$ 
18. Khan MA, Patel PG, Ganesh AG, Rais N, Faheem SM, Khan ST. Assessing Methanogenic Archaeal Community in Full Scale Anaerobic Sludge Digester Systems in Dubai, United Arab Emirates. The open microbiology journal 2018;12:123.

19. Khan MA, Ashar NN, Ganesh AG, Rais N, Faheem SM, Khan ST. Bacterial Community Structure in Anaerobic Digesters of a Full Scale Municipal Wastewater Treatment Plant-Case Study of Dubai, United Arab Emirates. Journal of Sustainable Development of Energy, Water and Environment Systems 2019;7(3):385-98.

20. Claesson MJ, Wang Q, O'Sullivan O, Greene-Diniz R, Cole JR, Ross RP, et al. Comparison of two next-generation sequencing technologies for resolving highly complex microbiota composition using tandem variable 16S rRNA gene regions. Nucleic acids research 2010;38(22):e200-e.

21. Magoč T, Salzberg SL. FLASH: fast length adjustment of short reads to improve genome assemblies. Bioinformatics 2011;27(21):2957-63.

22. Edgar RC, Haas BJ, Clemente JC, Quince C, Knight R. UCHIME improves sensitivity and speed of chimera detection. Bioinformatics 2011;27(16):2194-200.

23. Yang Y, Yu K, Xia Y, Lau FT, Tang DT, Fung WC, et al. Metagenomic analysis of sludge from full-scale anaerobic digesters operated in municipal wastewater treatment plants. Applied microbiology and biotechnology 2014;98(12):5709-18.

24. Sundberg C, Al-Soud WA, Larsson M, Alm E, Yekta SS, Svensson BH, et al. 454 pyrosequencing analyses of bacterial and archaeal richness in 21 full-scale biogas digesters. FEMS microbiology ecology 2013;85(3):61226.

25. Regueiro L, Veiga P, Figueroa M, Alonso-Gutierrez J, Stams AJ, Lema JM, et al. Relationship between microbial activity and microbial community structure in six full-scale anaerobic digesters. Microbiological research 2012;167(10):581-9.

26. Su X-L, Tian Q, Zhang J, Yuan X-Z, Shi X-S, Guo R-B, et al. Acetobacteroides hydrogenigenes gen. nov., Sp. nov., An anaerobic hydrogen-producing bacterium in the family Rikenellaceae isolated from a reed swamp. International journal of systematic and evolutionary microbiology 2014;64.

27. Praveckova M, Brennerova MV, Holliger C, De Alencastro F, Rossi P. Indirect Evidence Link PCB Dehalogenation with Geobacteraceae in Anaerobic Sediment-Free Microcosms. Frontiers in microbiology 2016;7:933.

28. Petriglieri F, Nierychlo M, Nielsen PH, Mcllroy SJ. In situ visualisation of the abundant Chloroflexi populations in full-scale anaerobic digesters and the fate of immigrating species. PLoS One 2018;13(11):e0206255.

29. Gagliano MC, Braguglia CM, Petruccioli M, Rossetti S. Ecology and biotechnological potential of the thermophilic fermentative Coprothermobacter spp. FEMS Microbiology Ecology 2015;91(5).

30. Patel BKC, Morgan HW, Daniel RM. Fervidobacterium nodosum gen. nov. and spec. nov., a new chemoorganotrophic, caldoactive, anaerobic bacterium. Archives of Microbiology 1985;141(1):63-9.

31. Sekiguchi Y, Yamada T, Hanada S, Ohashi A, Harada H, Kamagata Y. Anaerolinea thermophila gen. nov., sp. nov. and Caldilinea aerophila gen. nov., sp. nov., novel filamentous thermophiles that represent a previously uncultured lineage of the domain Bacteria at the subphylum level. International journal of systematic and evolutionary microbiology 2003;53:1843-51.

32. Khan ST, Nakagawa Y, Harayama S. Sediminibacter furfurosus gen. nov., sp. nov. and Gilvibacter sediminis gen. nov., sp. nov., novel members of the family Flavobacteriaceae. Int J Syst Evol Microbiol 2007;57(Pt 2):265-9.

33. Dawoud M, Mulla MM. Environmental Impacts of Seawater Desalination: Arabian Gulf Case Study. Int J Environ Sustain 2012;1:22-37.

Page $12 / 19$ 
34. Paul P, Al Tenaiji AK, Braimah N. A Review of the Water and Energy Sectors and the Use of a Nexus Approach in Abu Dhabi. Int J Environ Res Public Health 2016;13(4):364-.

35. Inoue K, Ono H, Abe-Yoshizumi R, Yoshizawa S, Ito H, Kogure K, et al. A light-driven sodium ion pump in marine bacteria. Nature communications 2013;4:1678.

36. FitzGerald JA, Allen E, Wall DM, Jackson SA, Murphy JD, Dobson ADW. Methanosarcina Play an Important Role in Anaerobic Co-Digestion of the Seaweed Ulva lactuca: Taxonomy and Predicted Metabolism of Functional Microbial Communities. PLoS One 2015;10(11):e0142603-e.

37. Ito T, Yoshiguchi K, Ariesyady HD, Okabe S. Identification of a novel acetate-utilizing bacterium belonging to Synergistes group 4 in anaerobic digester sludge. ISME J 2011;5(12):1844-56.

38. Zellner G, Alten C, Stackebrandt E, Conway de Macario E, Winter JJAoM. Isolation and characterization of Methanocorpusculum parvum, gen. nov., spec. nov., a new tungsten requiring, coccoid methanogen. 1987;147(1):13-20.

39. Kölbl D, Pignitter M, Somoza V, Schimak MP, Strbak O, Blazevic A, et al. Exploring Fingerprints of the Extreme Thermoacidophile Metallosphaera sedula Grown on Synthetic Martian Regolith Materials as the Sole Energy Sources. 2017;8(1918).

40. Sakai S, Imachi H, Hanada S, Ohashi A, Harada H, Kamagata Y. Methanocella paludicola gen. nov., sp. nov., a methane-producing archaeon, the first isolate of the lineage 'Rice Cluster l', and proposal of the new archaeal order Methanocellales ord. nov. 2008;58(4):929-36.

41. Kessler PS, Daniel C, Leigh JA. Ammonia switch-off of nitrogen fixation in the methanogenic archaeon Methanococcus maripaludis: mechanistic features and requirement for the novel GInB homologues, Nifl1 and Nifl2. Journal of Bacteriology 2001;183(3):882-9.

\section{Figures}

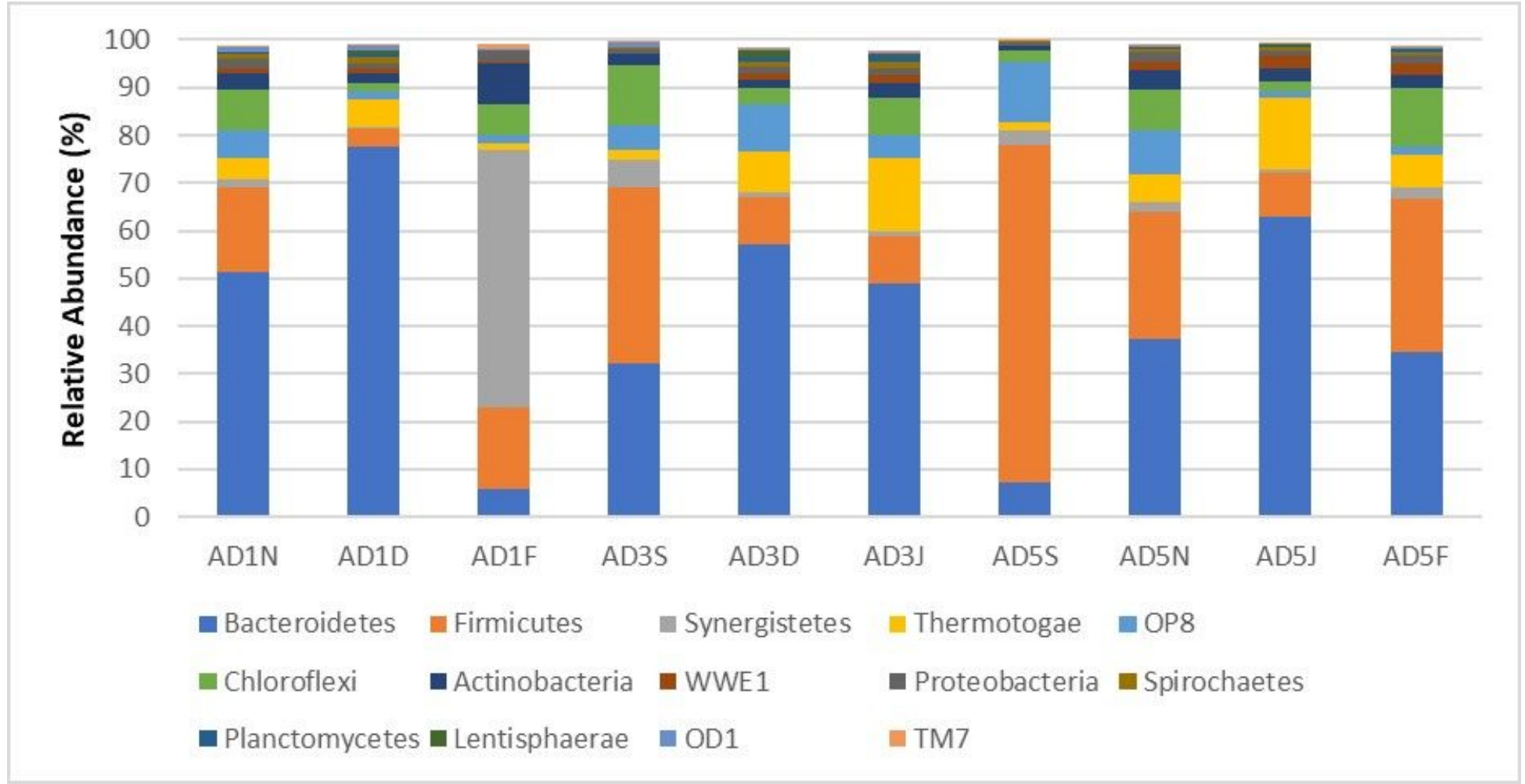


Figure 1

The abundant phyla of bacterial community across all three anaerobic digester samples

A

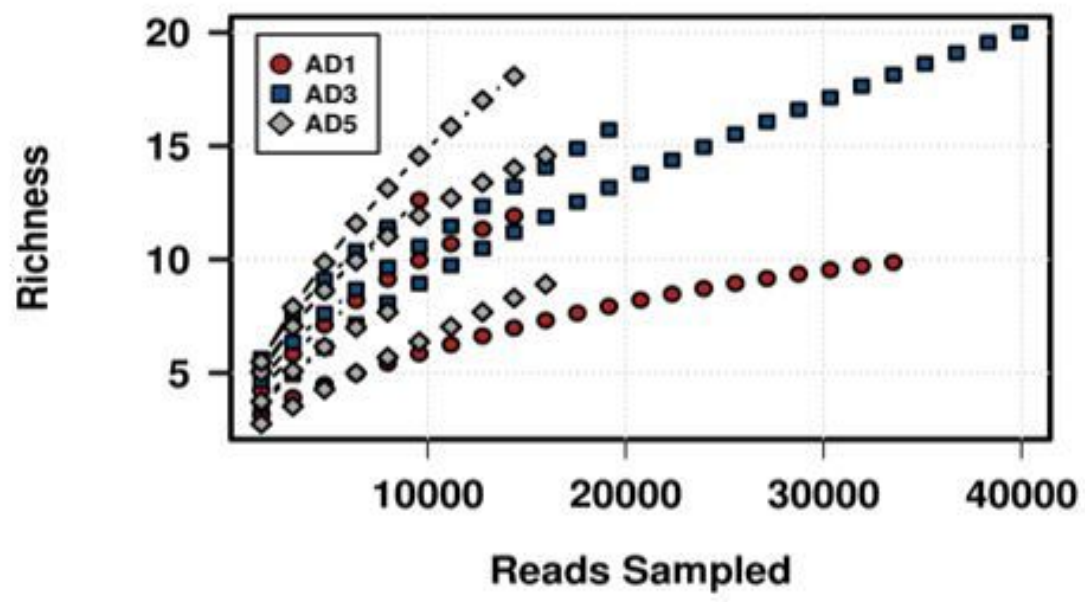

B
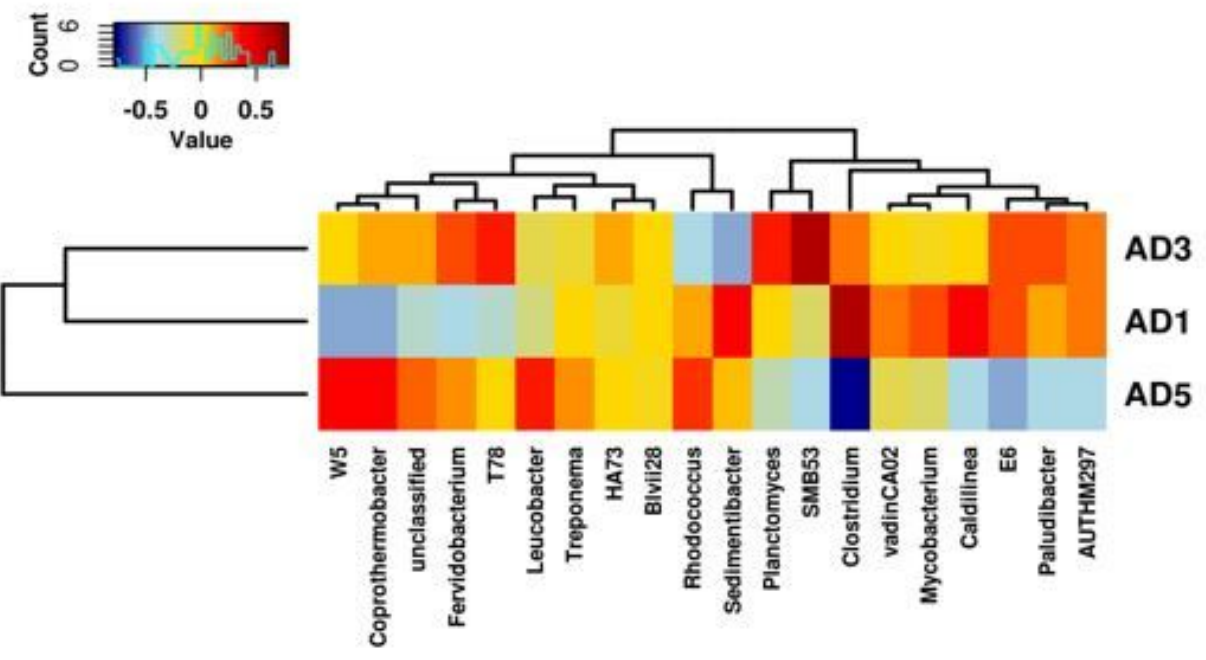

Figure 2

Panel A shows rarefaction curves of bacterial species diversity in the three anaerobic digesters (AD1, AD3 and AD5). Species were defined based on 3\% difference in the sequence. Three samples from each digester were chosen. While panel B shows heat map of hierarchical clustering of twenty genera with the highest mean relative abundance across the three anaerobic digesters 


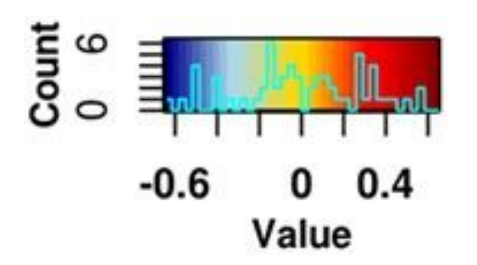


A

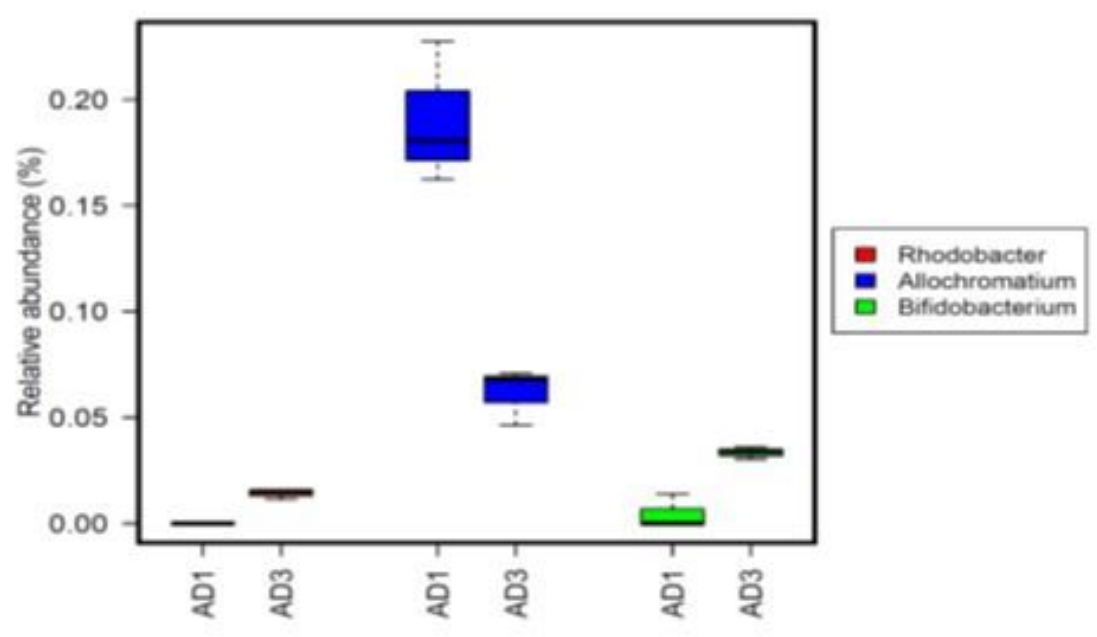

B

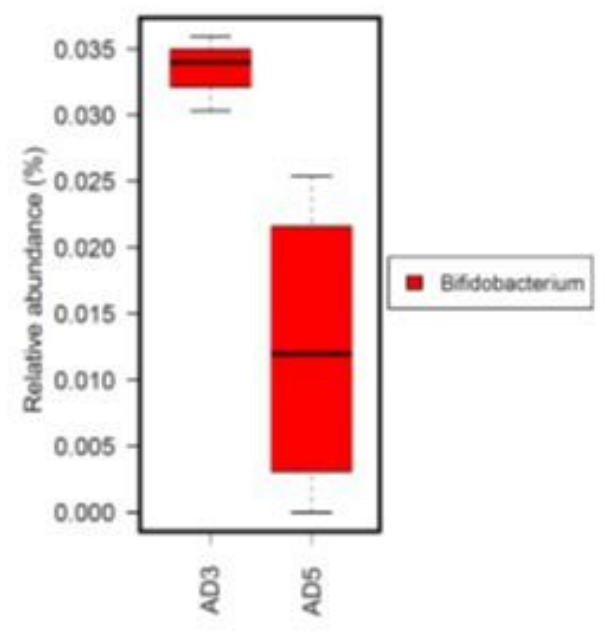

C

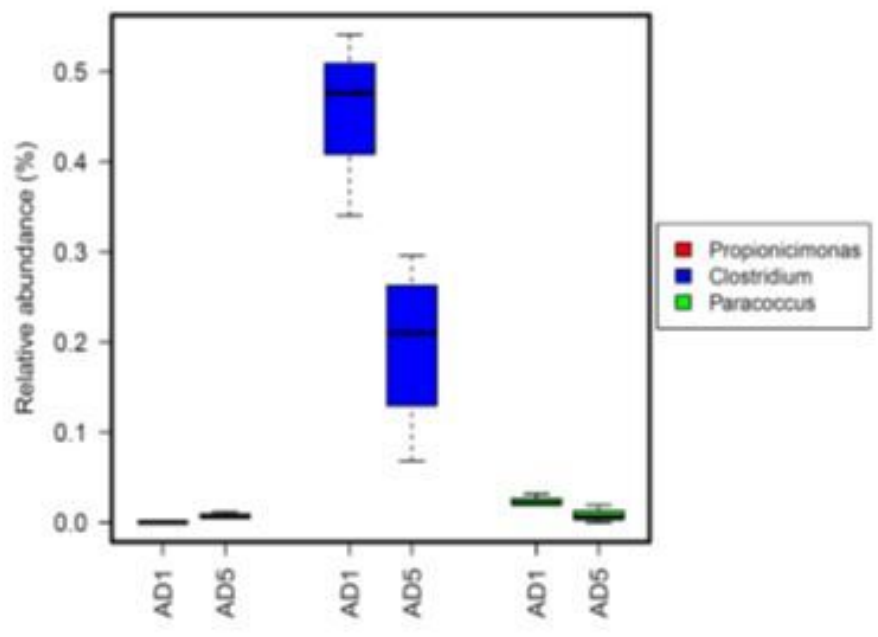

\section{Figure 4}

Inter group comparison for relative abundance of differentially present genera (A) AD1 v/s AD3 (B) AD3 v/s AD5 (C) AD1 v/s AD5 


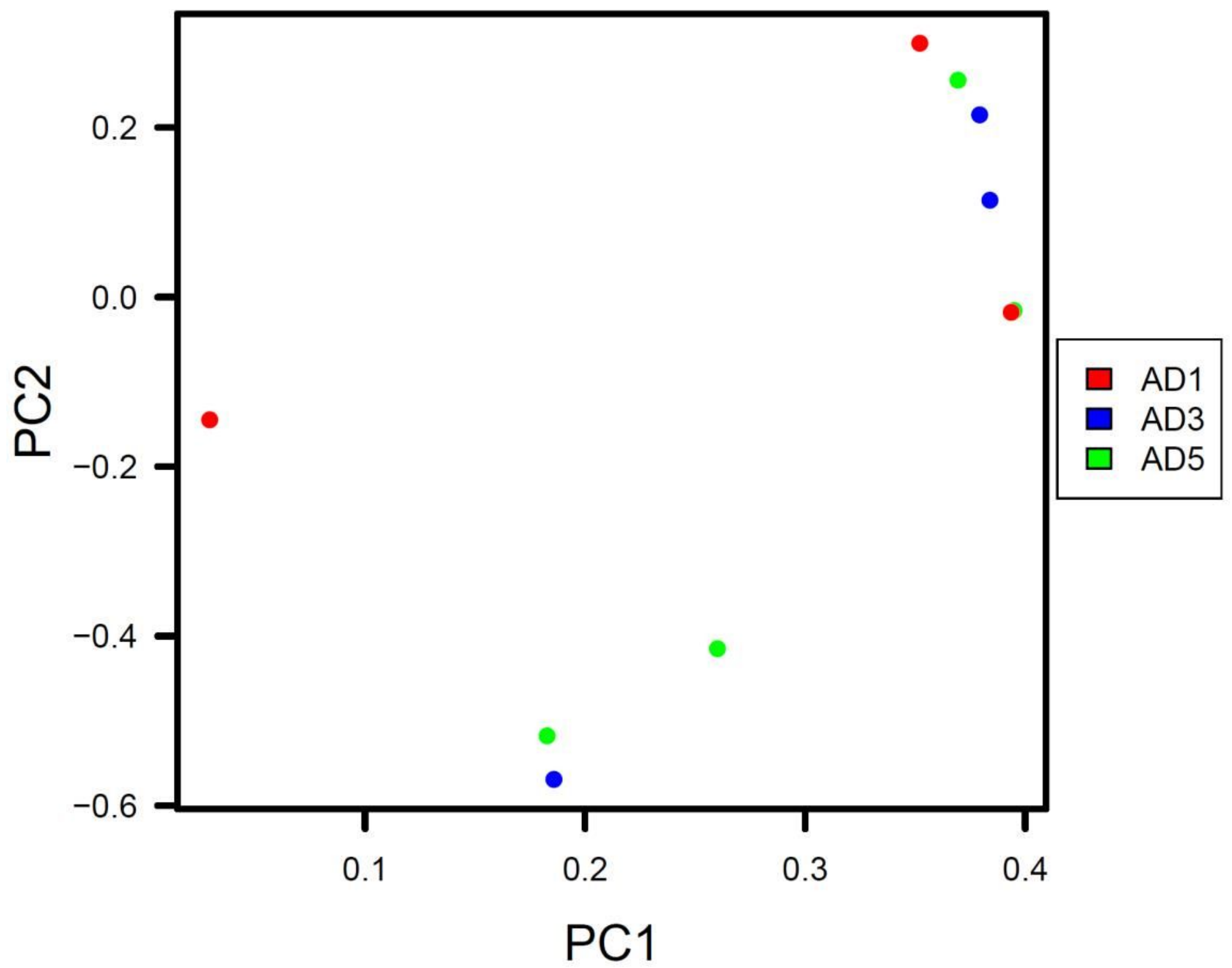

Figure 5

PCA plot for different groups at genera level 
A

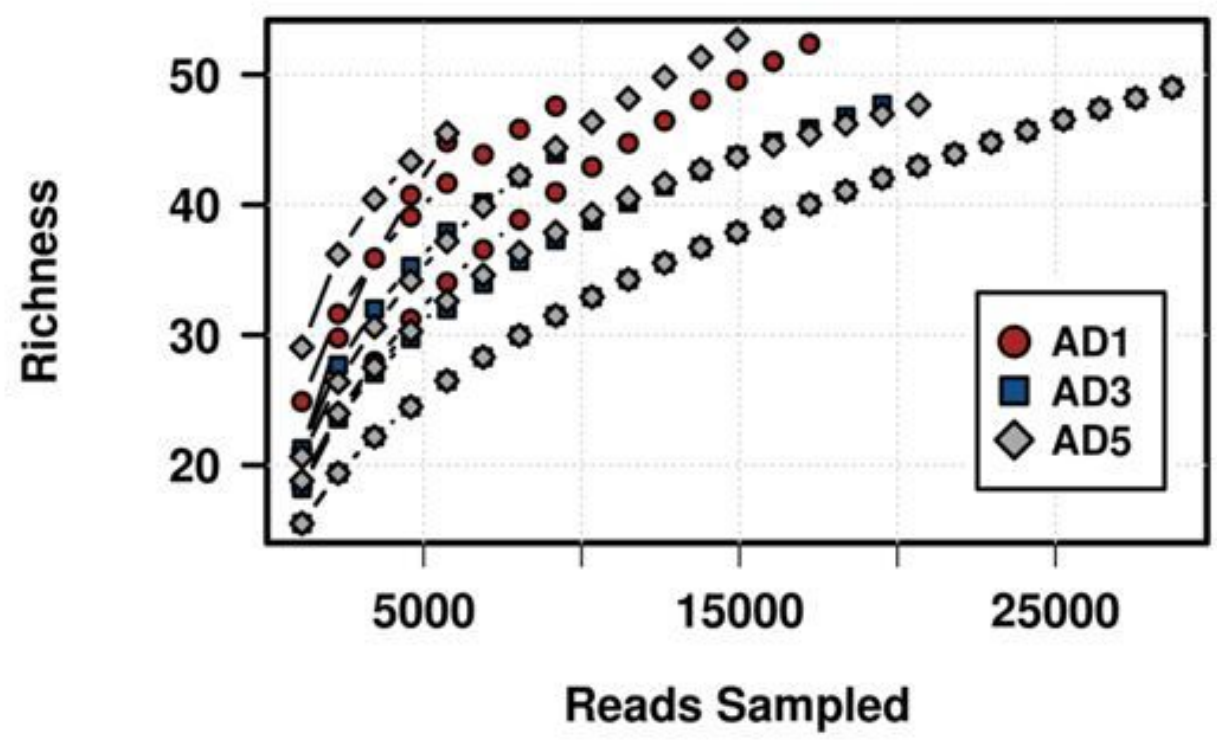

B

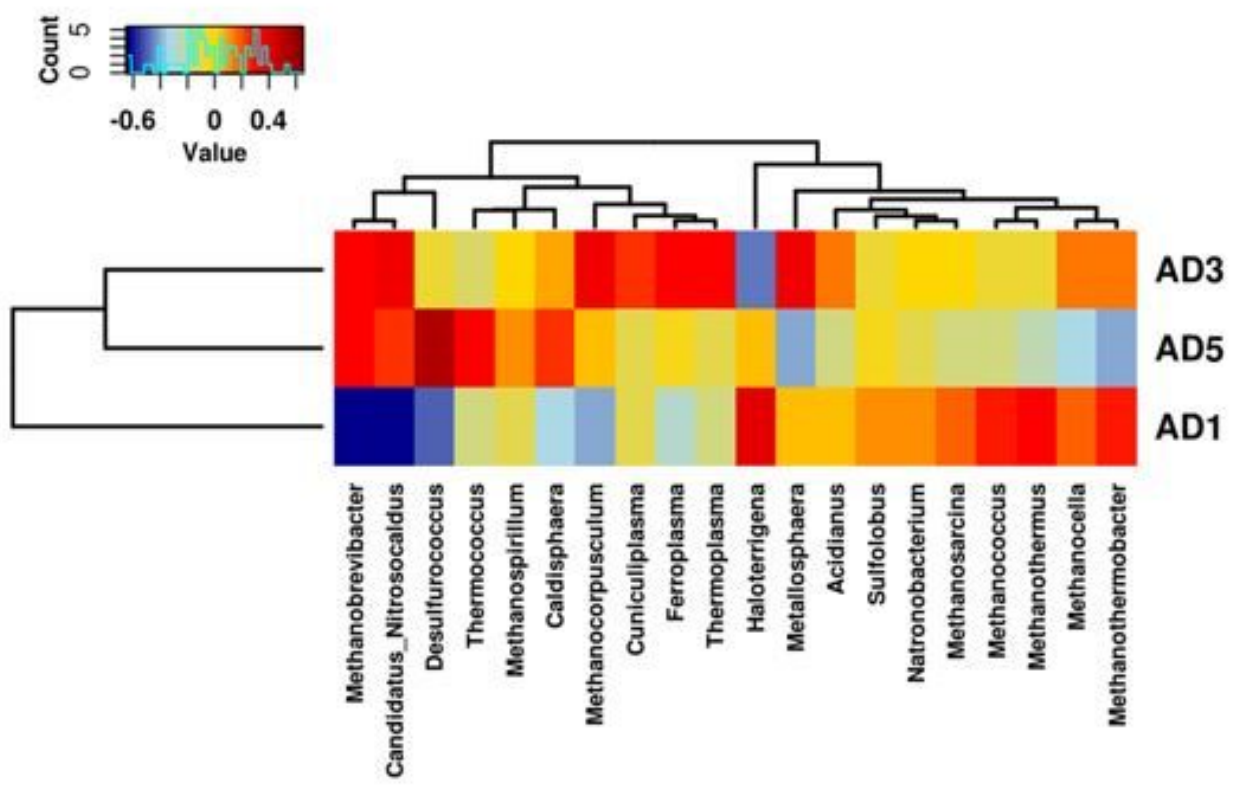

Figure 6

Panel $A$; rarefaction curves showing diversity of Archaea species in the three anaerobic digesters (AD1, AD3 and AD5). A 3\% difference in the sequence was used to define a species. Three samples from each digester were chosen. Panel B shows heat map of hierarchical clustering of twenty archaeal genera with the highest mean relative abundance across the three anaerobic digesters 


\begin{tabular}{|c|c|c|c|c|}
\hline Major taxa & AD1 & AD3 & AD5 & Process/products \\
\hline Bacteroidetes & 44.86 & 45.95 & 35.49 & \multirow{5}{*}{$\begin{array}{l}\text { Macromolecule } \\
\text { degradation } \\
\text { VFA \& Alcohols }\end{array}$} \\
\hline Firmicutes & 12.97 & 18.88 & 34.69 & \\
\hline Synergistetes & 18.67 & 2.77 & 1.98 & \\
\hline Thermotogae & 3.87 & 8.69 & 7.34 & \\
\hline Proteobacteria & 1.87 & 1.24 & 1.31 & \\
\hline Acetobacteroides (Blvii28) & 15.83 & 15.35 & 14.68 & \\
\hline Fervidobacterium & 3.27 & 7.74 & 7.20 & \\
\hline Clostridium & 0.45 & 0.40 & 0.20 & \\
\hline Syntrophomonas & 0.03 & 0.02 & 0.03 & \\
\hline Paludibacter & 0.27 & 0.44 & 0.17 & \\
\hline Mycobacterium & 0.61 & 0.19 & 0.19 & Acetate, $\mathrm{H}_{2} \& \mathrm{CO}_{2}$ \\
\hline Methanocorpusculum & 0.80 & 0.85 & 0.86 & 1 \\
\hline Metallosphaera & 0.08 & 0.07 & 0.05 & \\
\hline Methanocella & 0.02 & 0.01 & 0.02 & \\
\hline Methanococcus & 0.01 & 0.01 & 0.01 & Methane \\
\hline
\end{tabular}

\section{Figure 7}

Abundant microbial genera predicted to be associated with different process in the anaerobic digestion

\section{Supplementary Files}

This is a list of supplementary files associated with this preprint. Click to download.

- Supplimentaryfile4AD5Intragroupcomparison.xlsx

- Supplimentaryfile3AD3Intragroupcomparison.xIsx

- Supplimentaryfile2AD1Intragroupcomparison.xlsx

- Supplimentaryfile1Intercomparisonsgrp.xIsx

- SupplementaryFigures.docx 